\title{
Establishing neuronal circuitry: Hox genes make the connection
}

\author{
James Briscoe $^{1}$ and David G. Wilkinson ${ }^{2}$ \\ Developmental Neurobiology, National Institute for Medical Research, Mill Hill, London, NW7 1AA, UK
}

The vertebrate nervous system is composed of a vast array of neuronal circuits that perceive, process, and control responses to external and internal cues. Many of these circuits are established during embryonic development when axon trajectories are initially elaborated and functional connections established between neurons and their targets. The assembly of these circuits requires appropriate matching between neurons and the targets they innervate. This is particularly apparent in the case of the innervation of peripheral targets by central nervous system neurons where the development of the two tissues must be coordinated to establish and maintain circuits. A striking example of this occurs during the formation of the vertebrate head.

The head is a complex and integrated organization of many tissues including the central and peripheral nervous system, skeletal and connective tissue, and muscle. In the branchial region of the head, assembling the distinct tissues involves the initial formation of several reiterated structures: hindbrain segments, epidermal placodes, cranial ganglia, ectodermal branchial arches, and endodermal pharyngeal pouches (McGinnis and Krumlauf 1992; Lumsden and Krumlauf 1996; Graham 2001). These structures have precise spatial relationships with each other, for example, in the projection of motor neuron $(\mathrm{MN})$ axons from specific hindbrain segments to muscles residing in specific branchial arches. This raises the question of how the generation of the MNs and peripheral target tissues is coordinated to allow the formation of the correct neuronal circuitry. A recent study by Arenkiel et al. (2004) has advanced our understanding of this issue and here we discuss these new data in the context of previous work. Important to this new work is the relationship between hindbrain segments and neural crest cells that migrate to populate the branchial arches.

During early development, a transient metameric division of the hindbrain takes place in which eight transverse contiguous segments termed rhombomeres (r1-r8) are formed (Lumsden and Krumlauf 1996). Once established, restrictions in cell movement between rhombo-

\footnotetext{
Correspondence

${ }^{1}$ E-MAIL james.briscoe@nimr.mrc.ac.uk; FAX 44-20-8816-2523.

${ }^{2}$ E-MAIL dwilkin@nimr.mrc.ac.uk; FAX 44-20-8816-2523.

Article and publication are at http://www.genesdev.org/cgi/doi/10.1101/ gad.1227004
}

meres maintain these partitions. Each rhombomere adopts unique cellular and molecular properties that appear to underlie the spatial organization of the generation of cranial motor nerves and neural crest cells. Moreover, the coordination of positional identity between the central and peripheral derivatives of the hindbrain may underlie the anatomical and functional registration between MNs, cranial ganglia, and the routes of neural crest migration. Cranial neural crest cells derived from the dorsal hindbrain migrate ventral-laterally as discrete streams adjacent to $\mathrm{r} 2, \mathrm{r} 4$, and $\mathrm{r} 6$ to populate the first three branchial arches (BA1-BA3), respectively, where they generate distinct skeletal and connective tissue components and contribute to cranial ganglia (Köntges and Lumsden 1996; Le Douarin and Kalcheim 1999; Santagati and Rijli 2003). In the central nervous system, the generation of neurons is also segmentally organized. Among the first sets of neurons to form are the MNs of r2, r4, and r6, which will innervate the first three branchial arches as the trigeminal, facial, and glosspharyngeal nerves, respectively (Lumsden and Keynes 1989). Thus, hindbrain segmentation organizes multiple craniofacial elements, so that there is a correlation in the migration of neural crest cells and, at later stages, axons of MNs from hindbrain segments to branchial arches.

An important clue as to how the organization of multiple tissues could be coordinated in the head came from expression studies of the Hox family of homeodomaincontaining transcription factors that regulate regional identity along the anteroposterior axis. In the mouse there are four clusters of Hox genes (HoxA-HoxD), and a remarkable aspect of Hox gene expression is the collinearity of regulation so that the gene order of a complex is transposed into the spatial and dynamic pattern of expression (Duboule and Dolle 1989; Graham et al. 1989). Thus, genes at the $3^{\prime}$-end of a complex are expressed earlier and in more anterior regions than genes toward the 5 '-end. In the hindbrain, Hox genes have overlapping segmental domains of expression, such that each hindbrain segment expresses a specific combination or "code" of Hox genes consistent with a potential role in specification of segmental anteroposterior identity (Wilkinson et al. 1989; Hunt et al. 1991). Intriguingly, for many of these Hox genes, there is a corresponding expression in hindbrain segments and in neural crest cells migrating from those segments (Hunt et al. 1991). 
This coordinated expression of Hox genes in the hindbrain and neural crest is under the control of distinct gene regulatory elements (Maconochie et al. 1999), and suggests that there is coordinated anteroposterior specification in hindbrain segments and derivative neural crest. Taken together with the stereotyped migration pattern of neural crest into the branchial arches, and evidence that neural crest has a dominant role in patterning of muscles (Noden 1983), this suggests a transfer of positional information from the hindbrain to the branchial arches.

These findings suggest a model in which Hox genes specify anteroposterior identity in the hindbrain segments and in migrating neural crest, and contribute to a match in positional identity in the hindbrain and branchial arches that underlies the projection of MNs to appropriate muscle targets. Previous work has provided evidence for several aspects of this model.

\section{Hox genes pattern MNs and neural crest generated in rhombomere 4}

The generation of mouse mutants lacking individual or multiple Hox genes and the ectopic expression of Hox genes in chick embryos has provided important insights into the role these proteins play during hindbrain development. Perhaps best understood is the role of Hox genes in the development of $\mathrm{r} 4$ (Fig. 1A). This rhombomere generates the majority of the neural crest that contributes to the hyoid branchial arch (BA2; Köntges and Lumsden 1996). Also generated in $r 4$ are two distinct types of MNs: facial MNs that contribute to cranial nerve VII innervating muscles of the face and neck formed in BA2, and contralateral vestibuloacoustic (CVA) MNs of cranial nerve VIII that project contralaterally to the inner ear (Simon and Lumsden 1993; Auclair et al. 1996; Jacob and Guthrie 2000). Unlike other rhombomeres, $r 4$ does not produce reticulospinal serotonergic $(5 \mathrm{HT})$ neurons, and, instead, there is a prolonged period of MN generation (Pattyn et al. 2003). Hoxa1, Hoxa2, and Hoxb1 play prominent roles in the development of $\mathrm{r} 4$ and its derivatives (Gavalas et al. 1997, 1998, 2003; Rossel and Capecchi 1999). Hoxa1 and Hoxb1 are transiently expressed at early stages in the spinal cord and hindbrain, with an anterior boundary at the $\mathrm{r} 3 / \mathrm{r} 4$ interface, Hoxb1 expression is maintained in $\mathrm{r} 4$ and neurogenic neural crest from $\mathrm{r} 4$, and Hoxa2 expression occurs up to the $\mathrm{r} 1 / \mathrm{r} 2$ boundary and in neural crest cells migrating from $\mathrm{r} 4$ into the second branchial arch (Hunt et al. 1991; Murphy and Hill 1991; Prince and Lumsden 1994). Targeted inactivation of each gene in mouse embryos results in a distinct subset of defects in $\mathrm{r} 4$ development.

In mice lacking Hoxa1, r4 is smaller than normal, the boundary between $\mathrm{r} 3$ and $\mathrm{r} 4$ is not formed, and most of the $r 5$ territory is lost, resulting in a single rhombomerelike structure in the place of $\mathrm{r} 4 / \mathrm{r} 5$ (Carpenter et al. 1993; Mark et al. 1993; Gavalas et al. 1998). As a consequence there is a dramatic reorganization of the cell types normally generated in $\mathrm{r} 4$; this severely compromises the generation of r4-derived neural crest cells, and in place of facial MNs, a population of neurons forms with some characteristics of $\mathrm{r} 2$ trigeminal MNs. These data suggest
Figure 1. (A) Expression of Hoxb1 (red shading) and Hoxa2 (blue shading) in hindbrain segments and neural crest that contribute to the hyoid branchial arch (BA2). The gray background indicates mesoderm in the branchial arch, which is initially in the core of the arch, and later forms an integrated organization with neuralcrest-derived skeletal components. Hoxa2 regulates the patterning of skeletal structures, which in turn control the pattern of muscles in BA2. Hoxb1 expression becomes restricted to $\mathrm{r} 4$ in the hindbrain, and occurs in neural crest migrating from $\mathrm{r} 4$. At later stages, Hoxb1 is expressed in facial MNs that project axons to muscles in BA2, and in neural-crest-derived glial cells associated with facial MN axons. $(B)$ Changes to the projection of facial MNs in different Hoxb1 mutants. In Hoxb1 mutants, both the MNs and neural crest derivatives of $\mathrm{r} 4$ lack Hoxb1 expression, and the MNs fail to project correctly and later die. In chimeras between wild-type and Hoxb1 mutant embryos, there is a cell-autonomous defect in the projection of mutant MNs, whereas wildtype neurons appear to project normally. In embryos in which Hoxb1 is deleted selectively in premigratory neural crest using a Floxed allele of Hoxb1 and Wnt1cre, facial MNs are specified normally but have defects in their branching pattern and later die.

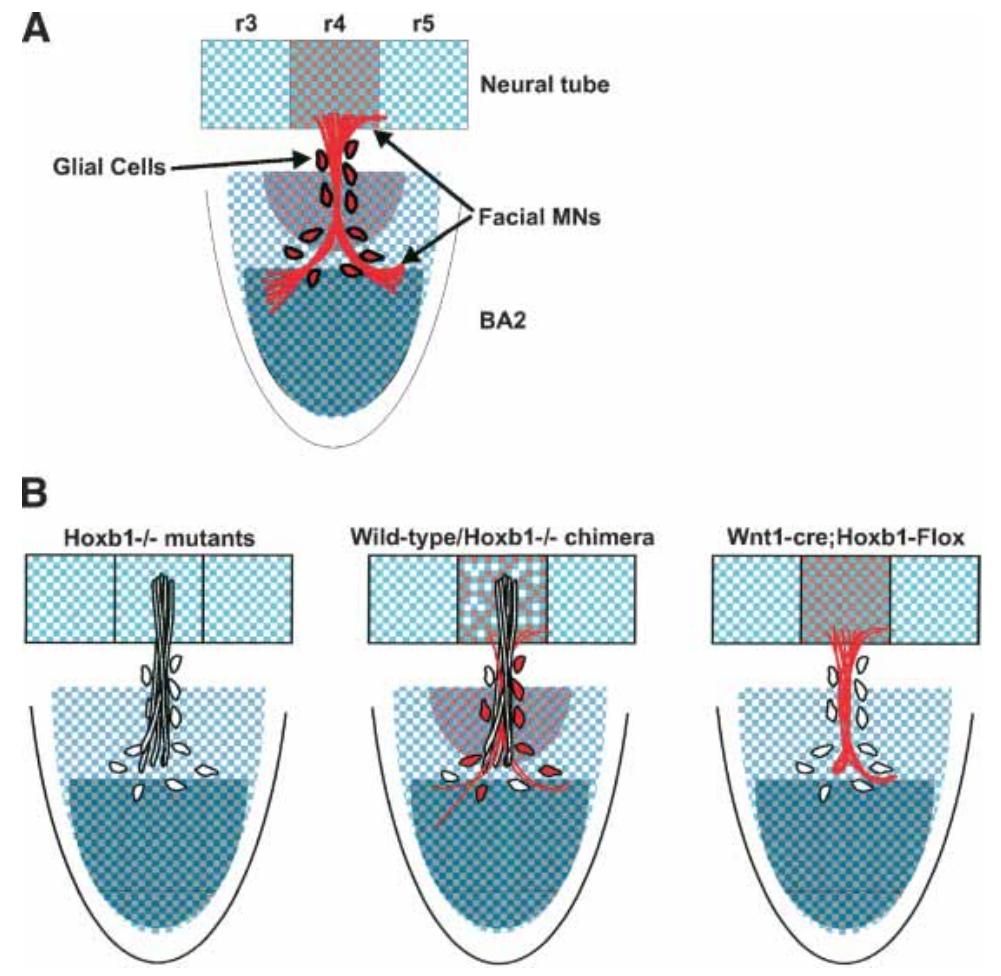


that Hoxa1 is important for specifying $\mathrm{r} 4$ identity and the cell types normally generated in this rhombomere. However, owing to the severity of the early defects in these mice, it is not known whether in addition to the requirement for Hoxal activity for the early specification of $\mathrm{r} 4$ segmental identity, Hox genes function selectively in later aspects of the development of r4-derived cell types.

In mice harboring a null mutation in Hoxa2, the initial establishment of $\mathrm{r} 4$ appears unaffected and facial and CVA MNs develop without apparent defects. In contrast, the neural crest of BA2 undergoes a dramatic homeotic transformation and, instead of generating structures appropriate for the hyoid arch, produces skeletal elements normally found in the BAl (Gendron-Maguire et al. 1993; Rijli et al. 1993; Gavalas et al. 1997). As a result, although facial MNs are specified and project axons out of the neural tube, by late stages of development the size of the facial nucleus and the calibre of the VII nerve is significantly reduced, presumably because misspecification of BA2 muscles results in the lack of neurotrophic support for facial MNs (Gavalas et al. 1997; F. Rijli, pers. comm.). The importance of Hoxa2 in the control of hyoid arch development has also been confirmed by gainof-function experiments in which the forced expression of Hoxa2 in chick or frog induces second arch elements where the first arch would normally form (Grammatopoulos et al. 2000; Pasqualetti et al. 2000).

Conversely, in Hoxb1-null mice there were no overt defects in $\mathrm{r} 4$ neural-crest-derived tissues, and BA2 formed normally; nor were defects apparent in the overall organization of $\mathrm{r} 4$. However, although MNs were generated in r4 of embryos lacking Hoxb1, facial and CVA neurons were incorrectly specified, and ectopic 5HT neurons were concomitantly produced in $\mathrm{r} 4$ (Goddard et al. 1996; Studer et al. 1996; Gaufo et al. 2000; Pattyn et al. 2003). The MNs generated in Hoxb1 $1^{-1-} \mathrm{r} 4$ failed to migrate to their proper positions, and their axons did not project to the appropriate peripheral targets, and, instead, these MNs acquired characteristics of $\mathrm{r} 2$ trigeminal MNs (Fig. 1B). As a consequence, there was a loss of $\mathrm{r} 4 \mathrm{MNs}$ and the VII motor nerve resulting in muscle atrophy and facial paralysis. These data suggest that Hoxb1 is critical for the specification of $\mathrm{r} 4 \mathrm{MN}$ subtype identity. Whether neural crest cells derived from $\mathrm{r} 4$ also require Hoxb1 expression for the correct development of $\mathrm{r} 4 \mathrm{MNs}$ could not be addressed, as the apoptotic elimination of the MNs produced in Hoxb1 mutants precluded analysis.

\section{Coordinated expression of Hox genes in MNs and neural crest}

Together these analyses of mouse mutants lacking individual Hox genes have revealed important roles for Hox proteins in controlling the development of the hindbrain and its neural crest derivatives. The studies did not, however, address whether coordinated Hox gene expression in the hindbrain and derivative neural crest is required to maintain registration between hindbrain MNs and their targets. Gain-of-function experiments in the chick have been begun to address this issue. Using a retroviral expression vector, Bell et al. (1999) ectopically expressed Hoxb1 in r2, anterior to its normal limit of expression. In these embryos, the global misexpression of Hoxb1 induced marker genes characteristic of $\mathrm{r} 4 \mathrm{MNs}$ in $\mathrm{r} 2$, and several of these MNs were observed projecting contralaterally in a manner similar to r4 CVA neurons. This suggested that expression of Hoxb1 respecifies r2 MN identity toward an $\mathrm{r} 4$ fate. However, $\mathrm{r} 4$ facial MNs normally project axons into BA2, whereas the majority of MNs in r2 of embryos transfected with Hoxb1 continued to send axons into BA1, and a small number of $\mathrm{MN}$ axons projected from $\mathrm{r} 2$ to $\mathrm{BA} 2$. Because the global misexpression strategy used in these experiments results in both CNS and neural crest cells expressing Hoxb1, the possibility arose that the projection of respecified $\mathrm{r} 2$ MNs into BAl was caused by matching ectopic expression in neural crest migrating into BA1. To test this, Bell et al. (1999) performed a series of transfections and transplantations. First, the basal plate from $\mathrm{r} 2$ of an embryo transfected with $H o x b 1$ was transplanted into an untransfected embryo, thus confronting Hoxb1-expressing MNs with Hoxb1-negative BA1. In these experiments, instead of projecting into BA1 the majority of axons from the Hoxb1 expressing $\mathrm{r} 2$ rerouted into BA2. This suggested that Hoxb1 has a cell-autonomous influence on the projections of MNs, impelling Hoxb1-expressing MNs to send axonal trajectories toward Hoxb1-expressing target tissue. Second, Hoxb1-expressing neural crest progenitors were transplanted to $\mathrm{r} 2$ so that Hoxb1expressing neural crest migrated into BA1. This challenged MNs that lack Hoxb1 with neural crest that expresses Hoxb1 and resulted in the axonal trajectories of the trigeminal nerve, normally generated in $\mathrm{r} 2$, being disrupted and truncated.

These data suggest that a mismatch between the Hox code present in MNs and that present in the periphery disrupts axonal pathfinding, raising the possibility that Hox genes direct the establishment of appropriate connectivity between neural tube and peripheral targets by regulating targeting mechanisms in both tissues. However, whether Hox gene expression is required for this remained to be examined. To address this question, Arenkiel et al. (2004) have now performed an elegant series of conditional genetic experiments in mouse to elucidate the roles of Hoxb1 in facial MNs and r4-derived neural crest.

\section{Hoxb1 is required in both MNs and neural crest for facial MN elaboration}

Arenkiel et al. (2004) initially confirmed that Hoxb1 is required cell-autonomously for the generation of facial MNs by generating chimeric embryos containing a mixture of wild-type and Hoxb1-null cells. In animals in which $\mathrm{r} 4$ contained a high contribution of $\mathrm{Hoxb1}^{-/-}$ cells, the facial nerve was absent, and the mice displayed facial paralysis (Fig. 1B). Examination of chimeric embryos confirmed that r4 MNs lacking Hoxb1 lacked the 
expression of markers normally associated with facial MNs, whereas $\mathrm{r} 4 \mathrm{MNs}$ generated from wild-type cells continued to express facial $\mathrm{MN}$ markers and projected axons into the VII nerve.

Although these data confirm the role of Hoxb1 in facial MN generation, they do not reveal whether Hoxb1 expression in neural crest cells is also required to establish the facial MN circuit. To address this issue, Arenkiel et al. (2004) adopted a conditional knockout strategy in which Hoxb1 was deleted in neural crest and its derivatives but not in MNs. To do this, a Hoxb1 allele flanked with LoxP sites was constructed, and two different Cre drivers were used to mediate recombination. To delete Hoxb1 from migrating neural crest, an AP2-cre driver was used. Experiments with a reporter line in which a marker gene was activated by Cre-mediated recombination indicated that AP2 functioned specifically in migrating neural crest, leaving Hoxb1 expression intact in the neural tube and premigratory neural crest. To remove Hoxb1 in neural crest prior to delamination from the neural tube, a Wnt1-cre driver was used. This driver exhibited a broader domain of recombination encompassing cells of the dorsal neural tube, premigratory neural crest, and neural crest derivatives, but leaving Hoxb1 expression intact in $\mathrm{r} 4 \mathrm{MNs}$.

Using appropriate crosses, the adult phenotypes of the conditional mutants in which Hoxb1 had been deleted using one of the two drivers were investigated. A striking difference was noted between deleting Hoxb1 with Wnt1-cre and AP2-cre. Removal of Hoxb1 after neural crest delamination, using the AP2-cre driver, did not affect the behavior of adult mice. In contrast, deletion of Hoxb1 from premigratory neural crest, using the Wnt1cre driver, resulted in a significant proportion of mutant animals displaying the same phenotype as animals completely lacking Hoxb1: Animals were unable to blink, retract their ears, or move their whiskers. Moreover, dissection of the face indicated that the branches of the facial nerve were missing and in the brainstem there was a loss of cell bodies where facial MNs would normally be expected. These data indicate that the expression of Hoxb1 is required in neural crest to establish or maintain the facial MN circuit (Fig. 1B). Moreover, the use of the two Cre deleter lines revealed the temporal window in which Hoxb1 is required, demonstrating that Hoxb1 function is required prior to neural crest delamination and emigration.

Not all animals harboring the conditional Hoxb1 allele and the Wnt1-cre driver displayed symptoms of facial paralysis. One explanation could be the inherent plasticity in neural crest identity and the potential for functional redundancy between Hox genes. Recent work has shown that the expression of Hox genes by migrating neural crest cells is not indicative of an irreversible commitment to a specific anteroposterior identity but, rather, a specification that subsequently requires signals from mesoderm in the branchial arches (Trainor and Krumlauf 2000; Schilling et al. 2001). Thus, in a proportion of animals, it is possible that these signals together with the expression of other Hox genes could allow the functional correction of facial MN elaboration. Alternatively, the stochastic nature of the timing of Cre-mediated recombination may result in the incomplete removal of Hoxb1 prior to the critical period in premigratory neural crest cells, thus allowing sufficient time for a pulse of Hoxb1 expression in some embryos.

Having established a role for Hoxb1 in $\mathrm{r} 4$ neural crest, Arenkiel et al. (2004) set out to determine at what point effects on facial MN development could be first identified. Examination of MN generation in conditional mutants lacking neural crest expression of Hoxb1 indicated that facial MNs are produced in normal numbers and the cell bodies migrate appropriately within the hindbrain neuroepithelium. By embryonic day 14.5 (E14.5), however, there began to be a noticeable decrease in the number of facial MNs. This coincides with the time at which limiting peripheral neurotrophic support initiates apoptotic pruning of MNs (Oppenheim et al. 2000). By E16.5, mutants harboring Wnt1-cre and the conditional Hoxb1 allele have only one-third of the number of facial MNs of wild-type littermates. These data suggested that the loss of Hoxb1 in neural crest affected facial MNs prior to E14.5 and point toward axon pathfinding defects. Consistent with this, although initial facial MN axonal projections were intact, decreased axon branching and fasciculation defects of the facial nerve could be detected by E12.5 in embryos in which Hoxb1 had been deleted from $\mathrm{r} 4$ neural crest.

These data raised the question of what type of Hoxb1expressing cells were responsible for the phenotype observed. Axons of facial MNs associate with cranial ganglia and fasiculate with the axons of sensory neurons and glia to form the VII nerve, which projects into the BA2 (Cordes 2001; Arenkiel et al. 2003). To address which of these cell types were derived from Hoxb1-expressing neural crest, a genetic lineage analysis was performed. Mice containing a Hoxb1-cre driver were crossed with a ROSA-26 GFP reporter line in which Cre-mediated recombination removed a stop cassette to allow GFP expression, thus indelibly marking cells descended from Hoxb1-expressing progenitors. Using this technique, the facial nerve was observed navigating a path to its targets, and examination of GFP expression with a series of markers indicated that only the glial cells associated with the facial nerve were descended from Hoxb1-expressing precursors. Other surrounding cells and target tissue lacked GFP expression, indicating that they were not the progeny of Hoxb1-expressing cells. Following the Hoxb1 lineage into postnatal animals demonstrated that Hoxb1-derived glial cells gave rise to $\sim 95 \%$ of the glial cells that ensheathed the facial nerve. These data suggested to Arenkiel et al. (2004) that it is the interaction between glial cells derived from Hoxb1-expressing precursors and the facial motor axons that is critical for the appropriate elaboration of the facial nerve.

What the defect is in the glial cells remains to be determined. Previous studies indicate that neural crest generation in Hoxb1-null animals is not obviously impaired (Goddard et al. 1996; Studer et al. 1996). Arenkiel et al. (2004) confirmed this finding using an explant as- 
say, which demonstrated by morphological criteria and expression of glial cell markers that there was no significant difference in glial generation between neural crest progenitors lacking Hoxb1 and wild-type progenitors. Thus, glial cell differentiation appears to remain intact in neural crest lacking Hoxb1, contrasting with genes such as Sox10 and ErbB3 that are required for generic glial cell development. Mice lacking either Sox10 or ErbB3 lack glial cells, and this results in severely compromised MN development and the eventual loss of MNs (Riethmacher et al. 1997; Britsch et al. 2001). The requirement for Hoxb1 in a later aspect of glial cell function is particularly intriguing given the expression profile and temporal requirement for Hoxb1 in $\mathrm{r} 4$ neural crest. Hoxb1 is expressed at early stages of neural crest specification and is down-regulated at later developmental points. Moreover, the effect of conditional deletion of Hoxb1 indicates that Hoxbl expression is required in premigratory neural crest. Thus, transient expression of Hoxb1 may impose a distinct anteroposterior identity on r4-derived peripheral glial cells that is required for the later maturation and maintenance of facial motor circuitry.

These findings raise several interesting questions. Given that facial MNs are lost at the time of normal pruning of these neurons, it is possible that glial cells provide a specific trophic support. Another possibility is that glial cells are required for initial steps of axon branching and pathfinding in order for the axons to reach their appropriate muscle targets, which provide survival factors. Insights into this are likely to come from identification of target genes of Hoxb1 in $r 4$ neural crest, and detailed analysis of the pathfinding of the facial nerve. Finally, it seems likely that the role of Hoxa2 in patterning the skeletal derivatives of $\mathrm{r} 4$ neural crest, which in turn patterns the muscles, could be required for later steps of facial MN pathfinding to specific muscle targets. The registration of positional identity underlying motor neuron circuitry could thus involve coordinated expression of multiple Hox genes in distinct populations of neural crest cells.

\section{References}

Arenkiel, B.R., Gaufo, G.O., and Capecchi, M.R. 2003. Hoxb1 neural crest preferentially form glia of the PNS. Dev. Dyn. 227: 379-386.

Arenkiel, B.R., Tvrdik, P., Gaufo, G.O., and Capecchi, M.R. 2004. Hoxb1 functions in both motoneurons and in tissues of the periphery to establish and maintain the proper neuronal circuitry. Genes \& Dev. 18: 1539-1552.

Auclair, F., Valdes, N., and Marchand, R. 1996. Rhombomerespecific origin of branchial and visceral motoneurons of the facial nerve in the rat embryo. J. Comp. Neurol. 369: 451461.

Bell, E., Wingate, R., and Lumsden, A. 1999. Homeotic transformation of rhombomere identity after localized Hoxb1 misexpression. Science 284: 2168-2171.

Britsch, S., Goerich, D.E., Riethmacher, D., Peirano, R.I., Rossner, M., Nave, K.A., Birchmeier, C., and Wegner, M. 2001. The transcription factor Sox10 is a key regulator of peripheral glial development. Genes \& Dev. 15: 66-78.
Carpenter, E.M., Goddard, J.M., Chisaka, O., Manley, N.R., and Capecchi, M.R. 1993. Loss of Hoxa-1 (Hox-1.6) function results in the reorganization of the murine hindbrain. Development 118: 1063-1075.

Cordes, S.P. 2001. Molecular genetics of cranial nerve development in mouse. Nat. Rev. Neurosci. 2: 611-623.

Duboule, D. and Dolle, P. 1989. The structural and functional organization of the murine HOX gene family resembles that of Drosophila homeotic genes. EMBO J. 8: 1497-1505.

Gaufo, G.O., Flodby, P., and Capecchi, M.R. 2000. Hoxb1 controls effectors of sonic hedgehog and Mash1 signaling pathways. Development 127: 5343-5354.

Gavalas, A., Davenne, M., Lumsden, A., Chambon, P., and Rijli, F.M. 1997. Role of Hoxa-2 in axon pathfinding and rostral hindbrain patterning. Development 124: 3693-3702.

Gavalas, A., Studer, M., Lumsden, A., Rijli, F., Krumlauf, R., and Chambon, P. 1998. Hoxa1 and Hoxb1 synergize in patterning the hindbrain, cranial nerves and second pharyngeal arch. Development 125: 1123-1136.

Gavalas, A., Ruhrberg, C., Livet, J., Henderson, C.E., and Krumlauf, R. 2003. Neuronal defects in the hindbrain of Hoxal, Hoxb1 and Hoxb2 mutants reflect regulatory interactions among these Hox genes. Development 130: 5663-5679. Epub 2003 Oct 1.

Gendron-Maguire, M., Mallo, M., Zhang, M., and Gridley, T. 1993. Hoxa-2 mutant mice exhibit homeotic transformation of skeletal elements derived from cranial neural crest. Cell 75: 1317-1331.

Goddard, J., Rossel, M., Manley, N., and Capecchi, M. 1996. Mice with targeted disruption of Hoxb1 fail to form the motor nucleus of the VIIth nerve. Development 122:32173228.

Graham, A. 2001. The development and evolution of the pharyngeal arches. J. Anat. 199: 133-141.

Graham, A., Papalopulu, N., and Krumlauf, R. 1989. The murine and Drosophila homeobox clusters have common features of organisation and expression. Cell 57: 367-378.

Grammatopoulos, G.A., Bell, E., Toole, L., Lumsden, A., and Tucker, A.S. 2000. Homeotic transformation of branchial arch identity after Hoxa2 overexpression. Development 127: 5355-5365.

Hunt, P., Gulisano, M., Cook, M., Sham, M., Faiella, A., Wilkinson, D.G., Boncinelli, E., and Krumlauf, R. 1991. A distinct Hox code for the branchial region of the vertebrate head. Nature 353: 861-864.

Jacob, J. and Guthrie, S. 2000. Facial visceral motor neurons display specific rhombomere origin and axon pathfinding behavior in the chick. J. Neurosci. 20: 7664-7671.

Köntges, G. and Lumsden, A. 1996. Rhombencephalic neural crest segmentation is preserved throughout craniofacial ontogeny. Development 122: 3229-3242.

Le Douarin, N.M. and Kalcheim, C. 1999. The neural crest, 2nd ed. Cambridge University Press, New York.

Lumsden, A. and Keynes, R. 1989. Segmental patterns of neuronal development in the chick hindbrain. Nature 337: 424428

Lumsden, A. and Krumlauf, R. 1996. Patterning the vertebrate neuraxis. Science 274: 1109-1115.

Maconochie, M., Krishnamurthy, R., Nonchev, S., Meier, P., Manzanares, M., Mitchell, P.J., and Krumlauf, R. 1999. Regulation of Hoxa2 in cranial neural crest cells involves members of the AP-2 family. Development 126: 1483-1494.

Mark, M., Lufkin, T., Vonesch, J.-L., Ruberte, E., Olivo, J.-C., Dolle, P., Gorry, P., Lumsden, A., and Chambon, P. 1993. Two rhombomeres are altered in Hoxa-1 mutant mice. Development 119: 319-338. 


\section{Briscoe and Wilkinson}

McGinnis, W. and Krumlauf, R. 1992. Homeobox genes and axial patterning. Cell 68: 283-302.

Murphy, P. and Hill, R. 1991. Expression of mouse labial-like homeobox-containing genes, Hox 2.9 and Hox 1.6, during segmentation of the hindbrain. Development 111: 61-74.

Noden, D. 1983. The role of the neural crest in patterning of avian cranial skeletal, connective, and muscle tissues. Dev. Biol. 96: 144-165.

Oppenheim, R.W., Houenou, L.J., Parsadanian, A.S., Prevette, D., Snider, W.D., and Shen, L. 2000. Glial cell line-derived neurotrophic factor and developing mammalian motoneurons: Regulation of programmed cell death among motoneuron subtypes. J. Neurosci. 20: 5001-5011.

Pasqualetti, M., Ori, M., Nardi, I., and Rijli, F.M. 2000. Ectopic Hoxa2 induction after neural crest migration results in homeosis of jaw elements in Xenopus. Development 127: 5367-5378.

Pattyn, A., Vallstedt, A., Dias, J.M., Samad, O.A., Krumlauf, R., Rijli, F.M., Brunet, J.F., and Ericson, J. 2003. Coordinated temporal and spatial control of motor neuron and serotonergic neuron generation from a common pool of CNS progenitors. Genes \& Dev. 17: 729-737.

Prince, V. and Lumsden, A. 1994. Hoxa-2 expression in normal and transposed rhombomeres: Independent regulation in the neural tube and neural crest. Development 120:911-923.

Riethmacher, D., Sonnenberg-Riethmacher, E., Brinkmann, V., Yamaai, T., Lewin, G.R., and Birchmeier, C. 1997. Severe neuropathies in mice with targeted mutations in the ErbB3 receptor. Nature 389: 725-730.

Rijli, F.M., Mark, M., Lakkaraju, S., Dierich, A., Dolle, P., and Chambon, P. 1993. A homeotic transformation is generated in the rostral branchial region of the head by disruption of Hoxa-2, which acts as a selector gene. Cell 75: 1333-1349.

Rossel, M. and Capecchi, M.R. 1999. Mice mutant for both Hoxal and Hoxb1 show extensive remodeling of the hindbrain and defects in craniofacial development. Development 126: 5027-5040.

Santagati, F. and Rijli, F.M. 2003. Cranial neural crest and the building of the vertebrate head. Nat. Rev. Neurosci. 4: 806818.

Schilling, T.F., Prince, V., and Ingham, P.W. 2001. Plasticity in zebrafish hox expression in the hindbrain and cranial neural crest. Dev. Biol. 231: 201-216.

Simon, H. and Lumsden, A. 1993. Rhombomere-specific origin of the contralateral vestibulo-acoustic efferent neurons and their migration across the embryonic midline. Neuron 11: 209-220.

Studer, M., Lumsden, A., Ariza-McNaughton, L., Bradley, A., and Krumlauf, R. 1996. Altered segmental identity and ab normal migration of motor neurones in mice lacking Hoxb-1. Nature 384: 630-634.

Trainor, P. and Krumlauf, R. 2000. Plasticity in mouse neural crest cells reveals a new patterning role for cranial mesoderm. Nat. Cell Biol. 2: 96-102.

Wilkinson, D.G., Bhatt, S., Cook, M., Boncinelli, E., and Krumlauf, R. 1989. Segmental expression of Hox-2 homoeoboxcontaining genes in the developing mouse hindbrain. Nature 341: 405-409. 


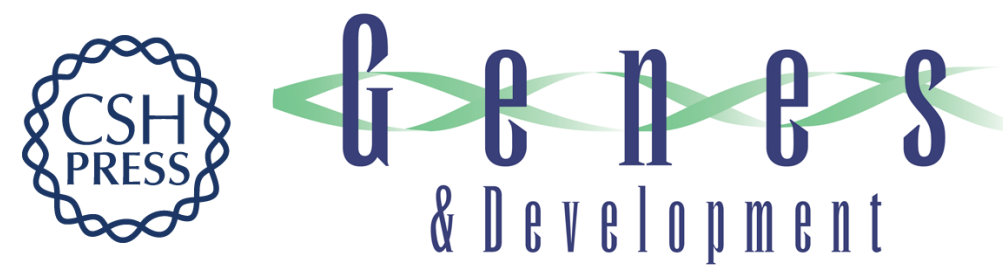

\section{Establishing neuronal circuitry: Hox genes make the connection}

\section{James Briscoe and David G. Wilkinson}

Genes Dev. 2004, 18:

Access the most recent version at doi:10.1101/gad.1227004

References This article cites 40 articles, 21 of which can be accessed free at: http://genesdev.cshlp.org/content/18/14/1643.full.html\#ref-list-1

\section{License}

Email Alerting Receive free email alerts when new articles cite this article - sign up in the box at the top Service right corner of the article or click here.

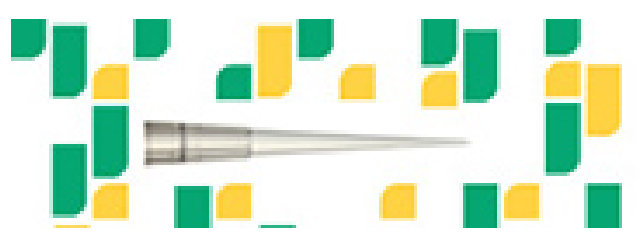

